\title{
Effects of Hormonal Contraceptives on Mood: A Focus on Emotion Recognition and Reactivity, Reward Processing, and Stress Response
}

\author{
Carolin A. Lewis ${ }^{1,2,3}$ - Ann-Christin S. Kimmig ${ }^{3,4} \cdot$ Rachel G. Zsido $^{1,2,5}$ - Alexander Jank ${ }^{6}$ Birgit Dernt $^{3,7,8}$. \\ Julia Sacher ${ }^{1,5,9}$
}

Published online: 7 November 2019

(C) The Author(s) 2019

\begin{abstract}
Purpose of Review We review recent research investigating the relationship of hormonal contraceptives and mood with a focus on relevant underlying mechanisms, such as emotion recognition and reactivity, reward processing, and stress response.

Recent Findings Adverse effects of hormonal contraceptives (HCs) on mood seem most consistent in women with a history of depressive symptoms and/or previous negative experience with HC-intake. Current evidence supports a negativity bias in emotion recognition and reactivity in HC-users, although inconsistent to some extent. Some data, however, do indicate a trend towards a blunted reward response and a potential dysregulation of the stress response in some HC-users.

Summary HC-effects on psychological and neurophysiological mechanisms underlying mood are likely context-dependent. We provide suggestions on how to address some of the contributing factors to this variability in future studies, such as HC-dose, timing, administration-mode, and individual risk. A better understanding of how and when HCs affect mood is critical to provide adequate contraceptive choices to women worldwide.
\end{abstract}

Keywords Hormonal contraceptives $\cdot$ Mood $\cdot$ Depression $\cdot$ Emotion $\cdot$ Reward $\cdot$ Stress

\section{Introduction}

With currently more than 100 million users worldwide [1], hormonal contraceptives (HCs) represent one of the most influential discoveries of the twentieth century [2]. HCs provide an effective option for contraception and safe family planning as well as for managing cycle-related physiological symptoms (e.g., ovulation pain, acne, hirsutism). Although this suggests that HC-use is beneficial for many women, there is a subset of women who suffer severe mood-related side effects. Thus,

This article is part of the Topical Collection on Reproductive Psychiatry and Women's Health

Carolin A. Lewis

lewis@cbs.mpg.de

1 Emotion Neuroimaging Lab, Max Planck Institute for Human Cognitive and Brain Sciences, Stephanstr. 1A, 04103 Leipzig, Germany

2 International Max Planck Research School on Neuroscience of Communication: Function, Structure, and Plasticity, Leipzig, Germany

3 Department of Psychiatry and Psychotherapy, University of Tuebingen, Calwerstr, 14, 72076 Tuebingen, Germany

4 International Max Planck Research School for Cognitive and Systems Neuroscience, University of Tuebingen, Tuebingen, Germany
Department of Neurology, Max Planck Institute for Human Cognitive and Brain Sciences, Leipzig, Germany

6 Department of Obstetrics, University Hospital Leipzig, Leipzig, Germany

7 Werner Reichardt Center for Integrative Neuroscience, University of Tuebingen, Tuebingen, Germany

8 LEAD Research School and Graduate Network, University of Tuebingen, Tuebingen, Germany

9 Clinic for Cognitive Neurology, University of Leipzig, Leipzig, Germany 
while substantial research has been dedicated to the physiological consequences of HC-use, such as cardiovascular risk, few studies have investigated the effects of HCs on mood and behavior.

Given that side effects such as depressive symptoms are typically reported as the main reason for discontinuing $\mathrm{HC}$-use $[3,4]$ and the relative scarcity of neuroimaging studies currently published in this area, additional research efforts to shed light on the neuropsychological side effects of HCs are warranted. With the emerging field of reproductive neuroscience, scientists are beginning to investigate the neural effects of $\mathrm{HC}$ use in humans. A better understanding of how HC-use influences mood may have a critical impact on translational psychiatry, considering that women are approximately twice as likely as men to develop depression [5] and ovarian hormonal fluctuations have been associated with depression susceptibility and prevalence in women [6]. Epidemiological data suggest that hormonal transition periods across the female lifespan, such as puberty, pregnancy and postpartum, and the perimenopause, are windows of heightened risk to develop depression [7•], comprising a possible reproductive subtype of depression [8]. Certain women are particularly susceptible to the subtle hormone fluctuations across the menstrual cycle, which may result in the development of premenstrual dysphoric disorder (PMDD) [9]. Given these reported associations between hormone fluctuations and depression susceptibility, and that HCs introduce synthetic ovarian hormones thereby modulating endogenous ovarian hormone production (for overview, see Fig. 1 and $[10,11,89,90])$, we review recent research investigating the relationship of HC-use and mood with a focus on relevant underlying mechanisms, such as emotion recognition and reactivity, reward processing, and stress responsivity.

Relying on Danish Registry data, Skovlund and colleagues $[12 \bullet \cdot]$ recently reported a link between antidepressant prescription and HC-use. The authors included data from more than one million women in the age of 15-34 years, who were using combined estradiol/progestin as well as progestin-only HCs in all available forms of administration (see Fig. 1 and $[10,11,89,90]$ for an overview of HC methods). In those women, risk ratios for first diagnoses of depression or first antidepressant-use increased during the first 6 months after initiation of HC-use (1.8-fold relative risk compared with naturally cycling women). Similarly, Zettermark and colleagues $[13 \bullet \bullet]$ investigated the prescription of psychotropic drugs (anxiolytics, hypnotics, sedatives, or antidepressants) within the first year of HC-use in a sample of 800,000 women from a Swedish health registry. Reported rates for psychotropic drug use indicated an adjusted odds ratio of 1.34 for a first-time psychotropic drug prescription in HC-users. However, both studies $[12 \bullet, 13 \bullet \cdot]$ were correlational in nature and reporting relative risks can be misleading as the incidence of these events is quite low [14]. While causation is not determinable in observational designs, both studies $[12 \bullet \bullet, 13 \bullet \bullet]$ investigated impressive sample sizes, providing essential epidemiological evidence to develop hypotheses for potential mechanisms underlying the reported associations of HC-use and depression risk.

Randomized, placebo-controlled trials (RCTs) represent the gold standard in intervention-based studies, in that they can provide the strongest possible evidence for causal effects. Several groups have now successfully applied this study design to investigate HC-effects on mood. Zethraeus and colleagues [15**] included over 300 women in a double-blind $\mathrm{RCT}$, testing the effect of a combined oral contraceptive (OC) versus placebo, on well-being and mood. Over the course of 3 months, women in the OC group reported significantly lower global scores on self-reported well-being compared with placebo, driven by the negative effect of OCs on scales measuring positive well-being, self-control, and vitality. However, mean depression scores did not differ significantly across groups and time points in self-reported Beck Depression Inventory (BDI) scores.

Another Swedish group took a more unconventional approach in their double-blind RCT: they aimed to sample participants more representative of $\mathrm{HC}$-users in the general population, thus deciding not to exclude women with previous or ongoing psychiatric disorders and respective medication, nor any women with a history of OC-use-associated onset of depressed $\operatorname{mood}[16 \cdot \bullet, 17 \cdot \bullet]$. In total, over 200 women participated in either a placebo or combined OC group for three treatment cycles. The authors reported small but significant mood-related adverse effects of OCs in self-reported anxiety, irritability, and mood swings. No significant effects of OCs were observed for the Montgomery-Asberg Depression Rating Scale. However, some women in the OC group also reported improvements in mood during the premenstrual phase of the cycle. Women with previous negative OCassociated experiences reported significantly more severe depressed mood after completion of the 3-month trial compared with women with no such history. A further aspect to consider is the effect of HCs on the expression of premenstrual mood symptoms. Here, one study reports no effect of HC-use on premenstrual mood (using a prospective cross-over design; [18]), while another study supports a beneficial association between HC-use and premenstrual mood symptoms (although cross-sectional; [19]).

In summary, the data currently available supports some mood-related side effects of $\mathrm{HC}$-use, most convincingly shown in women with a history of depressive symptoms. However, some women may experience beneficial effects of HC-use, specifically on premenstrual mood symptoms (see [20] for review). As HC-related side effects on mood are not 


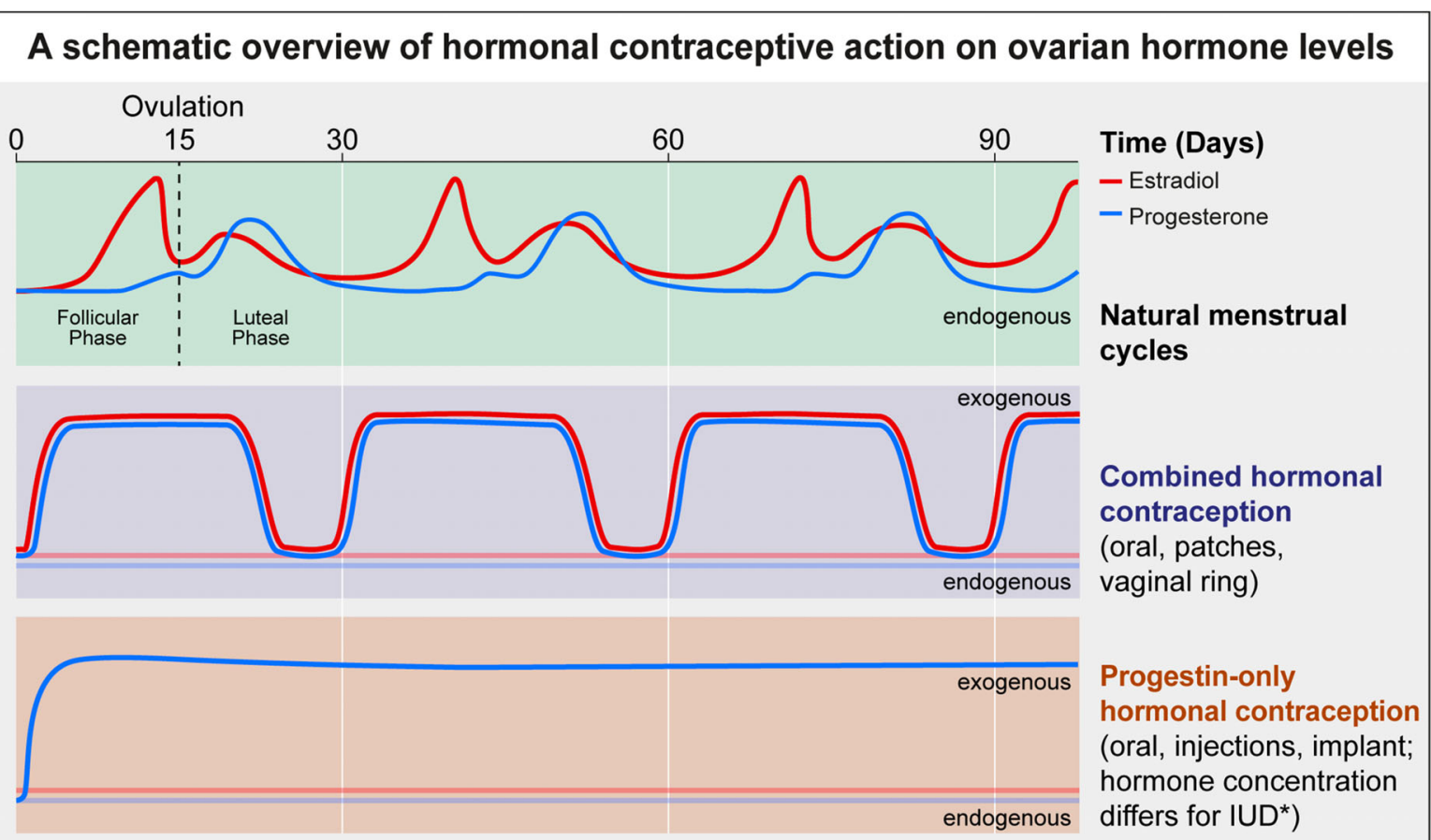

\section{Combined hormonal contraception}

Combined HCs contain a synthetic estrogen (ethinyl estradiol, EE) and a synthetic progesterone (progestin). All combined formulations generally work by inhibiting ovulation, thinning uterine lining, and thickening cervical mucus.

\section{Combined pill}

Daily intake of one pill, ideally at the same time each day. Combined pills are typically used in a $21 / 7$ regime, i.e. 21 days of pill-intake followed by 7 days of no intake or placebo-intake and period-type bleeding.

\section{Patch}

Thin, plastic patch that sticks to the skin and releases a daily dose of hormones through the skin into the bloodstream. The patch is placed on the lower abdomen, buttocks, outer arm, or upper body. Once a new patch is applied, it lasts for three weeks and is removed during the fourth week to allow for period-like bleeding.

\section{Vaginal ring}

Thin, flexible plastic ring, approximately 5 centimeters in diameter, which is inserted into the vagina, where it continually releases hormones for three weeks. It is removed during the fourth week and a new ring is inserted afterwards.

\section{Progestin-only hormonal contraception}

Progestin-only HCs interfere with ovulation and alter normal cyclical changes in the uterine lining, which may result in breakthrough bleeding. They also thicken cervical mucus, which prevents sperm from entering uterus and fallopian tube.

\section{Progestin-only pill}

Daily intake of one pill, ideally at the same time each day and without a break. Bleeding may become lighter, irregular, or more frequent.

\section{Injection}

Injection of a progestin (depot medroxyprogesterone acetate [DMPA]) in arm or buttocks once every 3 months. Bleeding may become more irregular, heavier, shorter, lighter, or stops.

\section{Intra-uterine device (IUD)}

Small, T-shaped device that is inserted into the uterus by a physician and can remain in place and function for up to 3-5 years. An IUD releases a progestin (levonorgestrel, LNG) into the uterus, which causes thickening of cervical mucus and thinning of uterine lining. Bleeding is reduced or stops completely. *In some women, IUD prevents ovulation, but most women continue to ovulate. Thus, endogenous hormone levels remain high enough to allow ovulation to take place.

\section{Implant}

Implantable plastic rod, which is matchstick-sized and flexible. A physician surgically inserts the rod under the skin of a woman's upper arm. The rod steadily releases a progestin into the bloodstream and can remain implanted for up to 3-5 years. It prevents ovulation, thins uterine lining, and thickens cervical mucus. Bleeding may become more irregular, heavier, shorter, lighter, or stops.
Fig. 1 Comparison of ovarian hormone profiles across the natural menstrual cycle (top row), and during intake of most common hormonal contraceptives, such as combined hormonal contraception (middle row), and progestin-only hormonal contraception (bottom row). The modes of action as well as intake characteristics of the most common hormonal contraceptives are described below. 
fully understood to date, additional research efforts to shed light on a possible impact of HCs on the mechanisms underlying mood regulation are warranted. Therefore, we review recent research on HC-effects on main psychological and neurophysiological mechanisms underlying mood regulation, such as the behavioral and neural correlates of emotion recognition and reactivity, reward processing, and stress response (Table 1).

\section{Influence of HCs on Psychological and Neurophysiological Mechanisms Underlying Mood Regulation}

\section{Emotion Recognition and Reactivity}

Negativity biases in key facets of emotion processing such as emotion recognition and emotional reactivity are thought to substantially contribute to the development and maintenance of depressed mood [43]. Mitigating negativity biases in emotion recognition and reducing emotional reactivity to negative stimuli can be effective strategies to improve mood [43-45].

The ability to correctly recognize emotional content from faces represents one major component of nonverbal communication [46], and impairments in this ability may play an important role in the development and maintenance of depressive symptoms [47, 48]. Several studies found impaired emotion recognition in OC-users [21,22], particularly for negative emotions [23-25], compared with naturally cycling women. For example, Pahnke and colleagues [21] report overall facial emotion recognition deficits in OC-users independent of emotional valence during the Reading-the-Mind-in-the-Eyes task, whereas Hamstra and colleagues [23, 24] identified a negativity bias in emotion recognition and emotional memory during a facial expression recognition task and an emotional categorization and memory task, respectively. Here, OC-users had significantly lower recognition accuracies for angry faces compared with naturally cycling women $[23,24]$. The authors further suggest that OC-users who are carriers of the mineralocorticoid receptor (MR) haplotype 1 or 3 have a more pronounced negativity bias, as these OC-users (1) had higher accuracy rates for detecting fearful and sad faces (unlike for angry faces), (2) had significantly longer reaction times for detecting these negative emotions, and (3) had better recall of negative characteristics in an emotional memory task, thus implicating an attention bias towards negative emotions [23]. Therefore, MR haplotype 1 or 3 carriers might be more vulnerable to depressogenic side effects of OCs than MR haplotype 2 carriers. Contrary to these findings, Radke and Derntl [26•] did not find evidence for an emotion recognition deficit in OC-users compared with naturally cycling women; however, they used only high-intensity emotional faces.
The current literature seems to confirm a negativity bias in emotion recognition in $\mathrm{HC}$-users, i.e., deficits in recognizing emotions accurately $[21,22,25]$ as well as an attentional bias to negative emotions [23-25]. However, emotion recognition abilities in HC-users seem to be affected by the task used in the study [25, 26.] or individual (epi-)genetic characteristics [22, 25, 23].

In addition to emotion recognition, emotional reactivity may also be linked to depressive symptoms. Emotional reactivity is the emotional response to an event, which can occur through multiple systems and differs in intensity and duration between individuals [49]. More intense and labile emotions, often accompanied by physiological arousal [50], have been associated with more depressive and internalizing symptoms $[51,52]$. While Radke and Derntl [26•] did not observe any differences in emotion recognition between OC-users and naturally cycling women, they reported that OC-users during the active OC-intake phase performed significantly better in an emotional reactivity task (affective responsiveness task) than OC-users during the pill-free week. Therefore, the active intake of OCs seems to be linked to an enhanced emotional reactivity towards positive as well as negative emotional scenarios. In line with these findings, a large-scale study recently showed that women using HCs showed significantly higher emotional reactivity by rating the valence of emotional stimuli more emotionally intense and recalling these emotional pictures significantly better than did naturally cycling women $[27 \bullet]$.

Neuroimaging research sheds further light on the possible modulatory effects of HCs on emotional reactivity. In a double-blind, placebo-controlled, functional magnetic resonance imaging (fMRI) study that only included women who had previously experienced OC-induced depressogenic side effects, Gingnell and colleagues [28] observed no behavioral differences between the OCassigned group and the placebo-assigned group in an emotional reactivity task (face-matching task with only negative faces) after 1 month of intake. The OC group did, however, show decreased habituation of the amygdala blood oxygenation level-dependent (BOLD) response compared with the placebo group. This finding could point towards a higher continued vigilance for negative emotional stimuli and therefore a biased attention towards negative stimuli in OC-users, possibly explaining adverse effects on mood. The OC group also showed reduced BOLD response of the left insula, the left middle frontal gyrus, and the bilateral inferior frontal gyri compared with the placebo group in response to negative emotional face stimuli [28]. However, these differences in BOLD response occurred in brain regions that are otherwise activated for positive or salient emotional stimuli. 


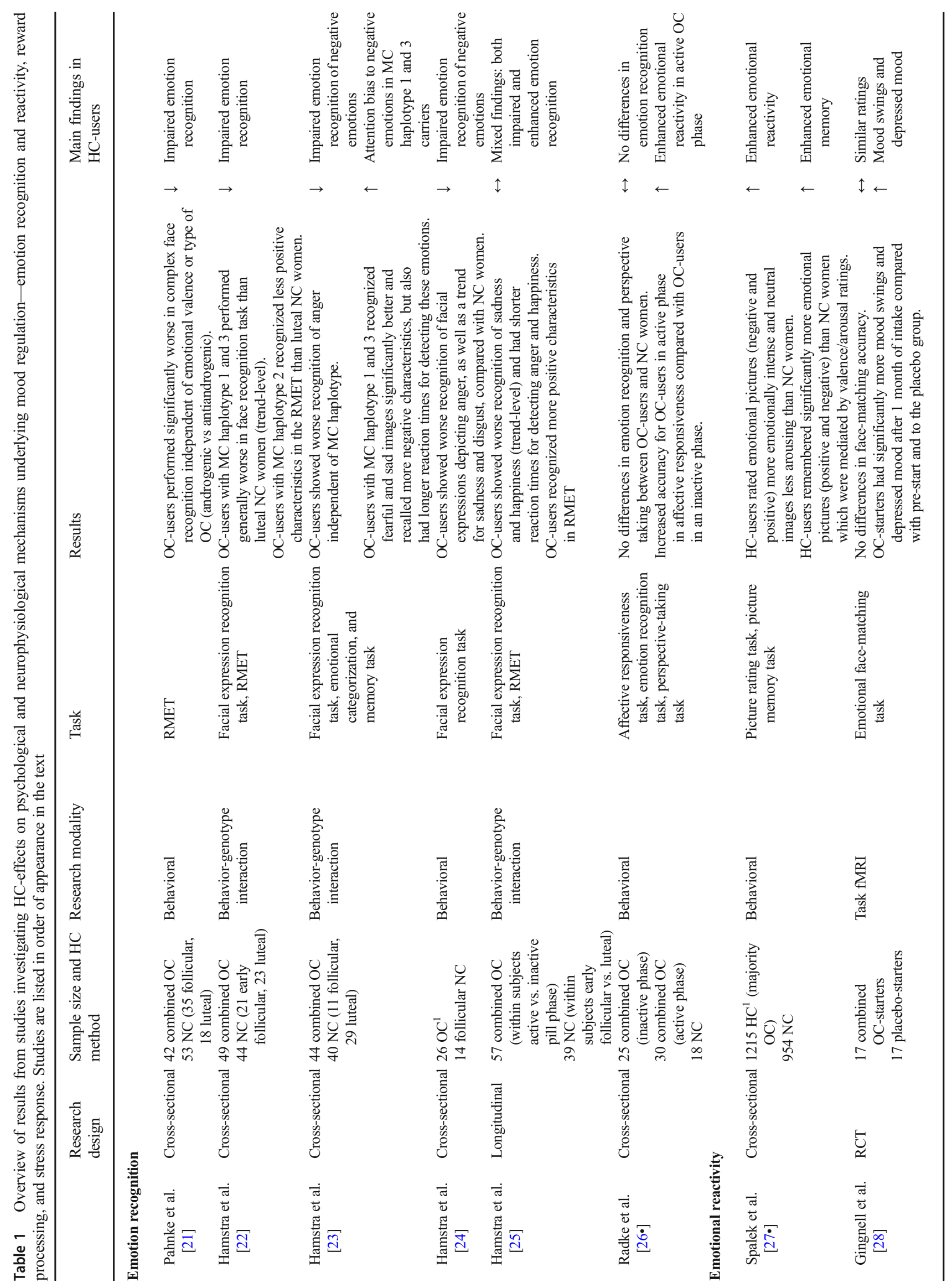




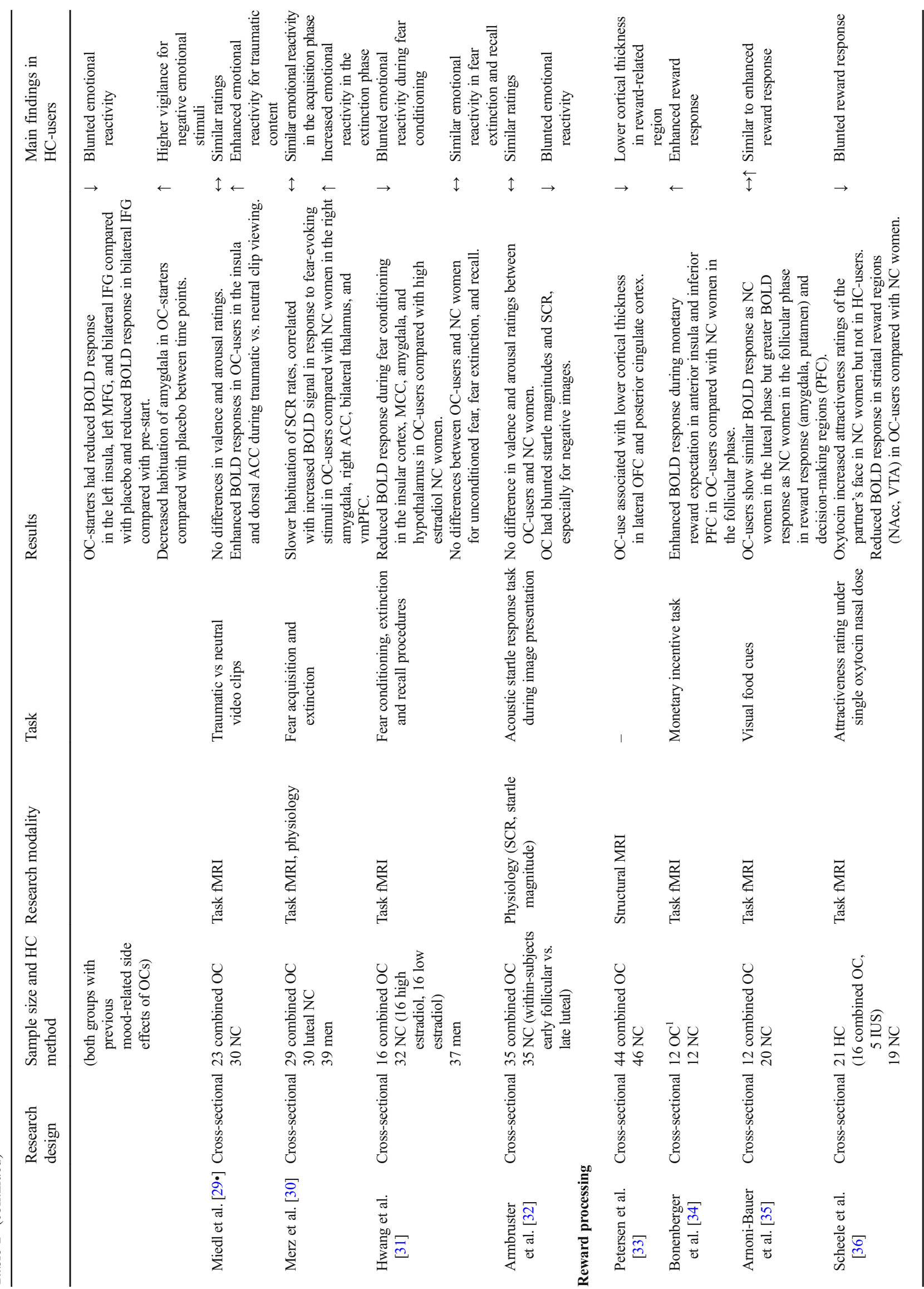




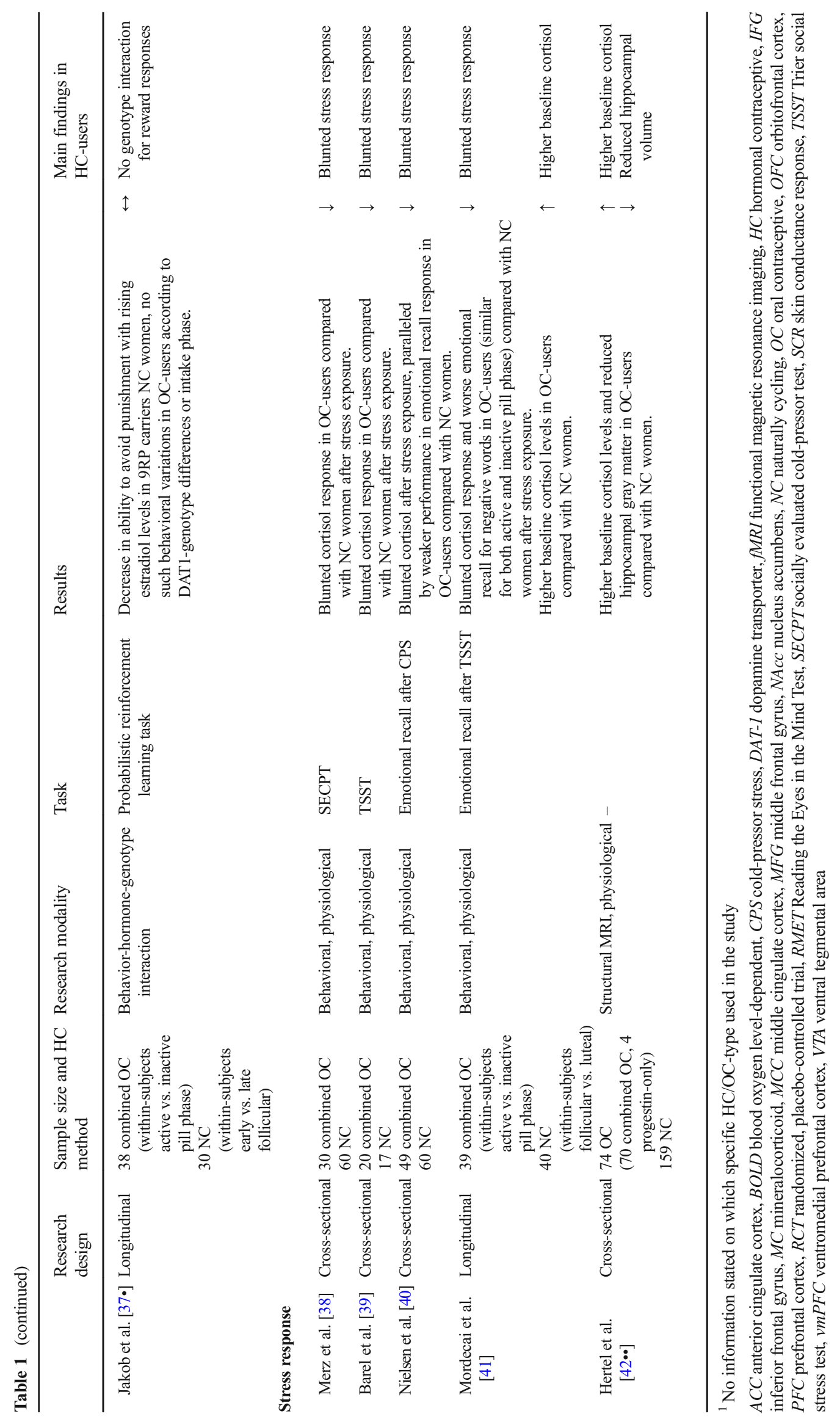


Specifically for fear processing, OC-induced effects on neural activation have been reported, such as enhanced activation of the fear network in OC-users compared with naturally cycling women, particularly in the insula and the dorsal anterior cingulate cortex (ACC) [29•]. These activation differences were independent of the valence and arousal ratings of the presented traumatic videos, which were similar between groups. Consistent with these findings, another study [30] found increased emotional arousal indicated by slower habituation of skin conductance response (SCR) rates, a physiological measure of the autonomic stress response, to be correlated with an increased BOLD signal in response to fear-evoking stimuli in OC-users compared with naturally cycling women. Group differences occurred in the right amygdala, right ACC, bilateral thalamus, and ventromedial prefrontal cortex (vmPFC). Unlike the previous study, Hwang and colleagues [31] did not observe any differences between OC-users and naturally cycling women in fear extinction but reported group differences for fear conditioning, i.e., reduced BOLD response of the fear network in OC-users compared with naturally cycling women. These neural correlates are further supported by physiological data, namely blunted SCR and startle reflex during fear conditioning in OC-users compared with naturally cycling women [32]. While emotional reactivity seems to be enhanced during fear extinction [30], neural [31] as well as physiological responses [32] are reduced during fear conditioning in OC-users.

Overall, the current data suggests a negativity bias in emotional reactivity shown by reduced BOLD responses to negative stimuli in brain regions that are otherwise relevant for processing salient and positive emotions [28], and enhanced BOLD responses in brain regions relevant for processing negative emotions, such as fear [29•,30]. These neuroimaging results are often not paralleled by behavioral outcomes $\left[26^{\bullet}\right.$, $28,29 \cdot, 32]$ and thus need to be interpreted with caution. However, as emotional reactivity occurs by definition through multiple systems [49], it might as well be that HC-use specifically impacts a very early stage of emotion processing, as reflected by $\mathrm{HC}$-induced modulation of emotional reactivity networks in the brain. On this account, further experimental designs including psychological, physiological, and neuroimaging measures when investigating HC-effects on emotional reactivity are highly encouraged.

\section{Reward Processing}

Recent models from computational psychiatry propose that negative mood may reflect the cumulative impact of differences between reward outcomes and expectations (e.g., [53, 54]). These models suggest a bidirectional interaction between mood and reward processing, which likely plays an important adaptive role in healthy behavior or, if compromised, could contribute to depressive disorders via a blunted hedonic response to rewards, i.e., anhedonia [55].

On a neural level, both endogenous estradiol and progesterone have neuroregulatory effects on the mesolimbic dopaminergic reward system [56-59]. In association with ovarian hormone fluctuations, changes in neural activation occur in the reward system [58], specifically in brain regions relevant for coding reward value and reward-expectancy such as the amygdala, the orbitofrontal cortex (OFC), and the striatum [60].

Literature on HC-related modulations of the reward system is relatively sparse. Petersen and colleagues [33] reported OCuse to be associated with significantly lower cortical thickness in the posterior cingulate cortex and the lateral OFC, with the latter revealing the most pronounced difference in cortical thickness between naturally cycling women and OC-users. This frontal cortex region is critical for the cognitive control of behavior, including response inhibition to stimuli with changing reward value [61]. Post hoc analyses suggest that these differences in cortical thickness were greater comparing OC-users and women in the follicular phase than comparing OC-users and women in the luteal phase. Yet, as this study used a cross-sectional design, we cannot infer causality nor establish a time-dependent association of OC-intake and OFC cortical thickness thus far.

OC-induced changes in brain morphology do not allow direct assumptions about behavioral changes, but taskbased fMRI studies can shed light on potential behavioral consequences. In a comparison of naturally cycling women with OC-users during a monetary incentive task, OCusers were more sensitive to monetary rewards and showed enhanced BOLD response during monetary reward expectation in the anterior insula and inferior prefrontal cortex (PFC) relative to naturally cycling women in the follicular phase [34]. Another study observed greater neural activation to visual food stimuli in OC-users than naturally cycling women during the follicular phase, but no group differences between OC-users and naturally cycling women during the luteal phase [35]. This difference in BOLD response during the follicular phase was observed in brain regions of the reward system (amygdala, putamen) as well as executive frontal areas (PFC). The authors proposed that comparable progesterone levels in OC-users and naturally cycling women in the luteal phase may underlie the similar BOLD responses between groups (similar to [33]). However, these studies were limited by their cross-sectional design and small sample sizes [34, 35] or lack of behavioral outcome measures [35]. Another study [36] included behavioral outcome measures and reported enhanced attractiveness ratings of the partner's face in naturally cycling women but not in HC-users after intranasal administration of oxytocin. The concomitant increased BOLD responses in nucleus accumbens (NAcc) and ventral tegmental area (VTA) were also more 
pronounced in the naturally cycling group than in the $\mathrm{HC}$ group. Taken together, task-based fMRI studies seem to provide rather mixed results, which could be due to the varying tasks used, e.g., investigating primary $[35,36]$ or secondary rewards [34]. Replication studies, preferably studies comparing performance in both primary and secondary reward tasks, are needed to further elucidate this issue.

Preclinical evidence suggests that endogenous estradiol levels can increase dopamine release in the reward system, specifically in the striatum $[62,63]$. Behavioral studies in humans partly support this finding as a positive correlation between endogenous estradiol levels and enhanced reward sensitivity in women, but paradoxically no increase in motivation for higher rewards from the early to the late follicular phase (i.e., with rising endogenous estradiol levels) have been reported [64]. Women have also been shown to be less sensitive for immediate rewards with rising estradiol levels from the early to the late follicular phase, but this effect was mainly driven by women with lower frontal dopamine levels (based on the COMT Met158Val polymorphism) [65]. These results nurtured the hypothesis of a hormone-genotype interaction, suggesting that particularly women with lower dopamine distribution would be affected by endogenous estradiol changes. Jakob and colleagues [37•] tested this hypothesis and investigated how endogenous estradiol levels and polymorphisms of the dopamine transporter (DAT1) interact. In this study, women performed a probabilistic feedback learning task twice: naturally cycling women once during the early (low estradiol) and subsequently during the late follicular phase (high estradiol) in comparison with OC-users once during active and once during inactive pill phase. Results indicated a significant effect of DAT1-genotype on reinforcement learning in naturally cycling women only, i.e., a decrease in the ability to avoid punishment with rising estradiol levels in 9RP carriers. The OC group did not show any such behavioral variations according to DAT1-genotype differences or intake phase. While these results suggest a small, dopamine-agonistic effect of endogenous estradiol on reward and punishment sensitivity (see [66] for an overview), the influence of $\mathrm{HC}$-induced changes in endogenous and exogenous estradiol levels on dopamine neurotransmission needs further research.

Overall, results from studies investigating the impact of HCs on reward processing are mixed (see Table 1): Studies have reported women on HCs to be more sensitive to rewards [34], to show comparable reward responses to naturally cycling women [35], or to experience blunted reward responses than naturally cycling women $[33,36,37 \cdot]$ as well as lower cortical thickness in brain regions of the reward system [33].
Based on the evidence currently available, the hypothesis of a blunted reward response in HC-users compared with naturally cycling women appears most supported, but remains to be systematically investigated.

\section{Stress Responsivity}

In women, high endogenous estradiol levels have been associated with an acutely blunted cortisol response, which is typically viewed as protective against acute psychosocial stress [67•]. A chronically blunted cortisol response, however, might increase the risk for depression. Atypical depression is characterized by hypoactivation of the hypothalamic-pituitary-adrenocortical (HPA) axis and describes a distinct pathophysiological phenotype, which is particularly common in women [6]. Recent work on the role of estradiol in the neural stress circuitry in women revealed increased BOLD response in the amygdala, hippocampus, and hypothalamus after a visual stress challenge in low endogenous estradiol states compared with high endogenous estradiol states (within-subject design, [68]). Notably, only healthy women demonstrated this endocrine regulation, while there was no evidence for this regulatory effect in women with recurrent depression in remission. This suggests a possible endocrine dysregulation associated with an altered stress response in women with depression (see also [69] for review).

HC-studies on stress responsivity using well-validated stress tasks consistently report a blunted cortisol response in OC-using women compared with naturally cycling women $[38,39]$. Nielsen and colleagues also found a blunted cortisol response in OC-users compared with naturally cycling women, paralleled by weaker performance for memorizing an emotional story: While naturally cycling women in the stress condition had enhanced recall for gist and detail, OC-users did not show such effects on memory [40]. Another study [41] extended these findings by showing that the blunted cortisol response previously reported in OC-users is similar during both the active and the inactive pill phase, following a psychosocial stress test (Trier social stress test, TSST). Interestingly, the authors also observed that OCusers had higher baseline salivary cortisol levels than naturally cycling women. Another study further substantiated this finding by investigating OC-related alterations in the HPA axis in OC-users compared with naturally cycling women $[42 \cdot \bullet]$ : The authors found overall elevated cortisol levels in OC-users as well as reduced hippocampal gray matter when investigating structural MRI scans. Given the evidence connecting chronic stress, elevated cortisol levels, and decreased hippocampal volume (e.g., [70]), these findings may indicate a potential protective effect of fluctuating endogenous estradiol levels through the mitigation of neurodegenerative effects of chronic stress on the hippocampus (see [67•] 
for review). The authors did not, however, find an association between cortisol levels and depressive symptoms (BDI scores) in OC-using women [42••]. Thus, the link between $\mathrm{HC}$-use, chronic stress, and depression susceptibility warrants further investigation [71].

Taken together, HC-intake seems to chronically alter HPA axis regulation, mirrored by (a) blunted cortisol responses after acute psychosocial and physical stressors [38-41] and (b) elevated baseline cortisol levels [41, 42••] (see Table 1). Further research is required to systematically address $\mathrm{HC}$-effects on the response to acute and chronic stress in different states of endogenous and exogenous ovarian hormones in health and disease to conclusively answer the question whether HC-effects on the stress response underlie mood-related $\mathrm{HC}$ side effects in women at risk.

\section{Summary and Future Directions}

In this review, we provide a summary of the most recent literature on HC-effects on women's mood, with a specific emphasis on some of the psychological and neurophysiological mechanisms that could underlie mood-related side effects of $\mathrm{HCs}$, which have been reported to occur in subgroups of women. We have reviewed the influence of HCs on emotion and reward processing as well as stress responsivity. We conclude that most of the reported results have yet to be replicated, thus no clear consensus can be reached based on these relatively heterogeneous datasets. From a methodological point of view, it is challenging to draw conclusions from neuroimaging results, which are not always paralleled by behavioral outcomes, and vice versa. Moreover, many neurobiological mechanisms are still not well understood. Given these limitations, most of the reported results have to be interpreted with caution, as the evidence is observational in most studies, and therefore, we cannot infer causality.

Many studies in this field only include women using OCs or women on different $\mathrm{HC}$ methods without stratification for each method. Consequently, the strongest conclusions can be drawn for OC-effects, as most of the available data include this $\mathrm{HC}$ method. Studies that did not stratify for different HC methods allow only for limited interpretation, as they contain different compounds and amounts of exogenous ovarian hormones, and also differ in the way of administration (see Fig. 1) and, thus, metabolization. Accumulating evidence [12••, 13••] suggests that different $\mathrm{HC}$ methods have divergent effects on mood: Non-oral HC methods (patch, vaginal ring, LNG-IUD) are more strongly associated with depression diagnosis or antidepressive treatment than OCs (combined OC and progestin-only OC). While these findings were correlational, some studies did take an interventional approach: Aleknaviciute and colleagues show that LNGIUD induced sensitized HPA axis responsivity on both an acute and a chronic stress parameter, compared with women taking combined OCs or naturally cycling women [72•]. However, there was no difference in depression scores 6 months after LNG-IUD insertion, but this study did not include a control group [73]. Vaginal ring contraception did not significantly modulate mood scores after 6 months of use [74, 75], and a systematic review found that vaginal ring users reported less depressive mood, irritability, and emotional liability than combined OCusers [76]. Concerning progestin-only HCs, a recent systematic review found only minimal association between progestin-only methods and validated depression measures [77•]. In summary, a direct comparison of different HC methods, ideally using a RCT, would add critical evidence to the current debate about potential negative side effects of HCs on mood. Such a systematic investigation could also provide insight regarding a more refined neurobiological understanding of how OCs may affect mood compared with other hormonal methods. Finally, this type of research would also have great clinical relevance by informing clinical recommendations for or against a specific $\mathrm{HC}$ method for a particular woman (for example, based on previous depression history).

A major methodological aspect that must be addressed but is rarely discussed or oversimplified concerns the way most neuroimaging studies use $\mathrm{HC}$-intake as a control variable for a low ovarian hormone state. This is only partly true. Indeed, the assessment of peripheral plasma levels of endogenous ovarian hormones, i.e., estradiol and progesterone, reveals low hormone levels. However, if a woman continuously takes exogenous ovarian hormones, either in an oral or non-oral route of administration, these exogenous ovarian hormones cross the blood-brain barrier [78, 79], a fact that should be considered in the interpretation of neuroimaging findings. Yet, this is only one aspect to consider: We do not yet know how a high exogenous and a low endogenous hormonal state interact, e.g., via feedback loops and cellular signaling, and how the neteffect of such an interaction can differ from a state of continuously fluctuating endogenous hormonal levels during the menstrual cycle. It is therefore challenging to interpret data from indirect neuroimaging modalities in vivo in the context of neurobiological mechanisms underlying the effects of HCs on behavior and brain function in women.

One technique that could provide essential insight into how $\mathrm{HC}$-induced hormonal states (i.e., low endogenous but high exogenous) may directly influence such neurobiological mechanisms in the brain is positron emission tomography (PET). Radioligand PET studies allow for the visualization and in vivo quantification of a specific neurochemical target at a specific molecular site [80] and thus could clarify the neurochemical changes accompanying $\mathrm{HC}$-use. We still require more tracer development dedicated to ovarian hormone receptors, but there are promising candidates. Ethinyl estradiol, the most commonly used synthetic estrogen in oral contraceptive formulations, is an estrogen receptor alpha $(\mathrm{ER} \alpha)$ agonist [81]. The tracer 
$16 \alpha-[18 \mathrm{~F}]$ fluoroestradiol-17 $\beta$ (FES) can be used to image ER $\alpha$, although FES is so far mostly used in clinical practice to assess breast cancer [82, 83] and needs further investigation for suitability of ER imaging in the human brain. Two FES-PET studies in female rats $[84,85]$ only observed specific binding in brain regions with high ER density (i.e., pituitary gland and hypothalamus). One FES-PET study [86] in a small, healthy, postmenopausal sample of women $(n=7)$ also found significant uptake in the pituitary, as well as in white matter, but administration of an ER antagonist only successfully reduced FES in the pituitary. In a recent review on sex hormones and available PET radiotracers [87], authors conclude that FES could be useful for assessing ER density in ER-dense brain regions but encourage development of novel PET tracers with higher affinity for further research. Progesterone receptor imaging can be done using the tracers 21-[18F]fluoro-16 $\alpha$-ethyl-19-norprogesterone (FENP), $21-[18 \mathrm{~F}]$ fluoro- $16 \alpha, 17 \alpha$-[(R)-(1'- $\alpha$-furylmethylidene)-dioxy]19-norpregn-4-ene-3,20-dione (FFNP), and the more metabolically stable 4-[18F]Fluoropropyl-Tanaproget (FPTP) [88], although this study [88] was performed in female rats in nonbrain areas (e.g., uterus and ovaries) and has yet to be studied in vivo in the human brain. Thus, while these tracers are informative of receptor density and occupancy, there is a critical need for development of radiotracers specifically dedicated to ovarian hormones, which could ultimately clarify how HCs modulate the delicate hormonal balance in the brain and thereby shed light on subsequent consequences for mood and depression susceptibility.

\section{Conclusion}

In order to advance our understanding of possible effects of HC-use on mood, we propose the following three perspectives to guide future research endeavors: (1) stratification for $\mathrm{HC}$ methods or direct comparison of the effects of different $\mathrm{HC}$ methods on mood, (2) initiation and implementation of rigorous RCT designs with adequate samples based on transparent a priori power-analyses, and (3) the development of quantitative methods to differentiate between exogenous and endogenous hormonal effects.

Based on the evidence currently available, it is likely that HC-intake can lead to mood-related side effects, particularly in women with a history of previous depressive episodes. Reported data indicate a trend towards negativity bias in emotion recognition and reactivity, a trend towards a blunted reward response and a potential dysregulation of the stress response in HC-users. Of note and not extensively discussed in this review, however, are the reported positive effects of $\mathrm{HC}$ use on mood in some women, especially for symptoms of PMDD (but see [20] for review). Any HC-effects on mood and the underlying psychological and neurophysiological mechanisms are therefore likely context-dependent.
It is imperative to take any reports on depressed mood as a potential side effect of $\mathrm{HC}$-intake seriously given the recent reports from large cohort studies $[12 \bullet \bullet, 13 \bullet \bullet]$ and the reality that discontinuation of HC-intake is most often motivated by such side effects, which can pose subsequent challenges in family planning $[89,90]$. In general, possible mood-related HC side effects should be carefully weighed against the profound benefit of $\mathrm{HC}$ methods for safe family planning. A better understanding of how and when HCs affect mood is of critical importance to provide adequate contraceptive choices to women worldwide.

Funding Information Open access funding provided by Max Planck Society. CAL, RGZ, and JS were supported by The Branco Weiss Fellowship - Society in Science, National Association for Research on Schizophrenia and Depression (NARSAD) Young Investigator Grant 25032 from the Brain \& Behavior Research Foundation, and by a Minerva Research Group grant from the Max Planck Society (all awarded to Dr. Sacher). ACK and BD were supported by the German Research Foundation, DFG (DE2319/9-1).

\section{Compliance with Ethical Standards}

Conflict of Interest The authors declare that they have no conflict of interest.

Human and Animal Rights and Informed Consent This article does not contain any studies with human or animal subjects performed by any of the authors.

Open Access This article is distributed under the terms of the Creative Commons Attribution 4.0 International License (http:// creativecommons.org/licenses/by/4.0/), which permits unrestricted use, distribution, and reproduction in any medium, provided you give appropriate credit to the original author(s) and the source, provide a link to the Creative Commons license, and indicate if changes were made.

\section{References}

Papers of particular interest, published recently, have been highlighted as:

- Of importance

- Of major importance

1. United Nations DoEaSA, Popluation Division. Trends in Contraceptive Use Worldwide 2015 (ST/ESA/SER.A/349). 2015.

2. Pletzer BA, Kerschbaum HH. 50 years of hormonal contraceptiontime to find out, what it does to our brain. Front Neurosci. 2014;8: 256. https://doi.org/10.3389/fnins.2014.00256.

3. Larsson G, Blohm F, Sundell G, Andersch B, Milsom I. A longitudinal study of birth control and pregnancy outcome among women in a Swedish population. Contraception. 1997;56(1):9-16.

4. Poromaa IS, Segebladh B. Adverse mood symptoms with oral contraceptives. Acta Obstet Gynecol Scand. 2012;91(4):420-7. https:// doi.org/10.1111/j.1600-0412.2011.01333.x.

5. Seedat S, Scott KM, Angermeyer MC, Berglund P, Bromet EJ, Brugha TS, et al. Cross-national associations between gender and mental disorders in the World Health Organization World Mental 
Health Surveys. Arch Gen Psychiatry. 2009;66(7):785-95. https:// doi.org/10.1001/archgenpsychiatry.2009.36.

6. Kuehner C. Why is depression more common among women than among men? Lancet Psychiatry. 2017;4(2):146-58. https://doi.org/ 10.1016/S2215-0366(16)30263-2.

7. Zsido RG, Villringer A, Sacher J. Using positron emission tomography to investigate hormone-mediated neurochemical changes across the female lifespan: implications for depression. Int Rev Psychiatry. 2017;29(6):580-96. https://doi.org/10.1080/09540261. 2017.1397607. Review and perspectives article, which provides a comprehensive overview on how hormone-mediated neurochemical changes may underlie increased depression risk in women and proposes guidelines on how to quantify such neurochemical changes in humans in vivo using neuroreceptor ligand positron emission tomography.

8. Baumeister H, Parker G. Meta-review of depressive subtyping models. J Affect Disord. 2012;139(2):126-40. https://doi.org/10. 1016/j.jad.2011.07.015.

9. Epperson CN, Steiner M, Hartlage SA, Eriksson E, Schmidt PJ, Jones I, et al. Premenstrual dysphoric disorder: evidence for a new category for DSM-5. Am J Psychiatry. 2012;169(5):465-75. https://doi.org/10.1176/appi.ajp.2012.11081302.

10. National Health Service. Which method of contraception suits me? 2019, January 3. https://www.nhs.uk/conditions/contraception/ which-method-suits-me/.

11. National Institutes of Health. What are the different types of contraception? 2017, January 31. https://www.nichd.nih.gov/health/ topics/contraception/conditioninfo/types.

12.• Skovlund CW, Morch LS, Kessing LV, Lidegaard O. Association of hormonal contraception with depression. JAMA Psychiatry. 2016;73(11):1154-62. https://doi.org/10. 1001/jamapsychiatry.2016.2387. Nationwide Danish prospective cohort study with a large sample size $(N=$ 1,061,997) combined data from the National Prescription Register and the Psychiatric Central Research Register to show that use of hormonal contraception was associated with subsequent prescription rates of antidepressants and first diagnosis of depression at a psychiatric hospital.

13.• Zettermark S, Perez Vicente R, Merlo J. Hormonal contraception increases the risk of psychotropic drug use in adolescent girls but not in adults: A pharmacoepidemiological study on 800000 Swedish women. PLoS One. 2018;13(3):e0194773. https://doi. org/10.1371/journal.pone.0194773. Nationwide Swedish prospective cohort study with a large sample size $(N=$ 815,662 ) that obtained information on drug prescriptions from the Swedish Prescribed Drug Register. They report that HC-use was associated with first-time psychotropic drug prescription within the first year of $\mathrm{HC}$-use.

14. Ross RA, Kaiser UB. Reproductive endocrinology: the emotional cost of contraception. Nat Rev Endocrinol. 2016;13(1):7-9. https:// doi.org/10.1038/nrendo.2016.194.

15.• Zethraeus N, Dreber A, Ranehill E, Blomberg L, Labrie F, von Schoultz B, et al. A first-choice combined oral contraceptive influences general well-being in healthy women: a double-blind, randomized, placebo-controlled trial. Fertil Steril. 2017;107(5):123845. https://doi.org/10.1016/j.fertnstert.2017.02.120. Randomized, placebo-controlled trial with a large sample size $(N=332)$, testing $\mathrm{HC}$-effects on well-being and mood. Over the course of three months, $\mathrm{OC}$-users reported lower well-being, self-control, and vitality compared with placebo; however, there were no differences in depression scores between groups.

16.• Bengtsdotter H, Lundin C, Gemzell Danielsson K, Bixo M, Baumgart J, Marions L, et al. Ongoing or previous mental disorders predispose to adverse mood reporting during combined oral contraceptive use. Eur J Contracept Reprod Health Care. 2018, 23(1): 45-51. https://doi.org/10.1080/13625187.2017.1422239.
Randomized, placebo-controlled trial with a large sample size $(N=202)$. Sample included women with previous or ongoing psychiatric disorders and respective medication, as well as women with a history of $\mathrm{OC}$-use-associated onset of depressed mood. They found that women with ongoing or previous disorders have increased risk for $\mathrm{OC}$-associated adverse mood effects.

17.• Lundin C, Danielsson KG, Bixo M, Moby L, Bengtsdotter H, Jawad I, et al. Combined oral contraceptive use is associated with both improvement and worsening of mood in the different phases of the treatment cycle-a double-blind, placebo-controlled randomized trial. Psychoneuroendocrinology. 2017;76:135-43. https://doi. org/10.1016/j.psyneuen.2016.11.033. Randomized, placebocontrolled trial with a large sample size $(N=202)$. Sample included women with previous or ongoing psychiatric disorders and respective medication, as well as women with a history of OC-use-associated onset of depressed mood. They found that women with ongoing or previous disorders have increased risk for $\mathrm{OC}$-associated adverse mood effects.

18. Ekenros L, Backstrom T, Hirschberg AL, Friden C. Changes in premenstrual symptoms in women starting or discontinuing use of oral contraceptives. Gynecol Endocrinol. 2019;35:1-5. https://doi. org/10.1080/09513590.2018.1534097.

19. Yonkers KA, Cameron B, Gueorguieva R, Altemus M, Kornstein SG. The influence of cyclic hormonal contraception on expression of premenstrual syndrome. J Women's Health (Larchmt). 2017;26(4):321-8. https://doi.org/10.1089/jwh.2016.5941.

20. Robakis T, Williams KE, Nutkiewicz L, Rasgon NL. Hormonal contraceptives and mood: review of the literature and implications for future research. Curr Psychiatry Rep. 2019;21(7):57. https://doi. org/10.1007/s11920-019-1034-z.

21. Pahnke R, Mau-Moeller A, Junge M, Wendt J, Weymar M, Hamm $\mathrm{AO}$, et al. Oral contraceptives impair complex emotion recognition in healthy women. Front Neurosci. 2018;12:1041. https://doi.org/ 10.3389/fnins.2018.01041.

22. Hamstra DA, de Kloet ER, Tollenaar M, Verkuil B, Manai M, Putman P, et al. Mineralocorticoid receptor haplotype moderates the effects of oral contraceptives and menstrual cycle on emotional information processing. J Psychopharmacol. 2016;30(10):1054 61. https://doi.org/10.1177/0269881116647504.

23. Hamstra DA, de Kloet ER, van Hemert AM, de Rijk RH, Van der Does AJ. Mineralocorticoid receptor haplotype, oral contraceptives and emotional information processing. Neuroscience. 2015;286: 412-22. https://doi.org/10.1016/j.neuroscience.2014.12.004.

24. Hamstra DA, De Rover M, De Rijk RH, Van der Does W. Oral contraceptives may alter the detection of emotions in facial expressions. Eur Neuropsychopharmacol. 2014;24(11):1855-9. https:// doi.org/10.1016/j.euroneuro.2014.08.015.

25. Hamstra DA, de Kloet ER, Quataert I, Jansen M, Van der Does W. Mineralocorticoid receptor haplotype, estradiol, progesterone and emotional information processing. Psychoneuroendocrinology. 2017;76:162-73. https://doi.org/10.1016/j.psyneuen.2016.11.037.

26. Radke S, Derntl B. Affective responsiveness is influenced by intake of oral contraceptives. Eur Neuropsychopharmacol. 2016;26(6): 1014-9. https://doi.org/10.1016/j.euroneuro.2016.03.004. The authors investigated $\mathrm{OC}$-effects on empathy using different paradigms, i.e., emotion recognition, perspective taking, and affective responsiveness. They report behavioral differences between OC-users in the active and inactive intake phase in emotional reactivity (tested in the affective responsiveness paradigm), which emphasizes a specific acute impact of OC-intake on behavioral performance.

27. Spalek K, Loos E, Schicktanz N, Hartmann F, de Quervain D, Stier $\mathrm{C}$, et al. Women using hormonal contraceptives show increased valence ratings and memory performance for emotional information. Neuropsychopharmacology. 2019. https://doi.org/10.1038/ 
s41386-019-0362-3. Study with a large sample size $(\boldsymbol{N}=\mathbf{2 1 6 9})$ showing that HC-users rated both negative and positive pictures more intense and had superior emotional memory than naturally cycling women. The association between $\mathrm{HC}$-use and memory performance was partially mediated by the differences in perceived emotional valence.

28. Gingnell M, Engman J, Frick A, Moby L, Wikström J, Fredrikson $\mathrm{M}$, et al. Oral contraceptive use changes brain activity and mood in women with previous negative affect on the pill - a double-blinded, placebo-controlled randomized trial of a levonorgestrel-containing combined oral contraceptive. Psychoneuroendocrinology. 2013;38(7):1133-44. https://doi.org/10.1016/j.psyneuen.2012.11. 006.

29. Miedl SF, Wegerer M, Kerschbaum H, Blechert J, Wilhelm FH. Neural activity during traumatic film viewing is linked to endogenous estradiol and hormonal contraception. Psychoneuroendocrinology. 2018;87:20-6. https://doi.org/10. 1016/j.psyneuen.2017.10.006. The authors studied peritraumatic processing in a fMRI paradigm, combined with valence and arousal ratings. OC-users had enhanced BOLD responses in areas of the fear processing network during watching traumatic film clips, but there were no differences in valence and arousal ratings between $\mathrm{OC}$-users and naturally cycling women.

30. Merz CJ, Tabbert K, Schweckendiek J, Klucken T, Vaitl D, Stark R, et al. Neuronal correlates of extinction learning are modulated by sex hormones. Soc Cogn Affect Neurosci. 2012;7(7):819-30. https://doi.org/10.1093/scan/nsr063.

31. Hwang MJ, Zsido RG, Song H, Pace-Schott EF, Miller KK, Lebron-Milad K, et al. Contribution of estradiol levels and hormonal contraceptives to sex differences within the fear network during fear conditioning and extinction. BMC Psychiatry. 2015;15:295. https://doi.org/10.1186/s12888-015-0673-9.

32. Armbruster D, Kirschbaum C, Strobel A. The not-so-bitter pill: effects of combined oral contraceptives on peripheral physiological indicators of emotional reactivity. Horm Behav. 2017;94:97-105. https://doi.org/10.1016/j.yhbeh.2017.06.009.

33. Petersen N, Touroutoglou A, Andreano JM, Cahill L. Oral contraceptive pill use is associated with localized decreases in cortical thickness. Hum Brain Mapp. 2015;36(7):2644-54. https://doi.org/ 10.1002/hbm.22797.

34. Bonenberger M, Groschwitz RC, Kumpfmueller D, Groen G, Plener PL, Abler B. It's all about money: oral contraception alters neural reward processing. Neuroreport. 2013;24(17):951-5. https:// doi.org/10.1097/WNR.0000000000000024.

35. Arnoni-Bauer Y, Bick A, Raz N, Imbar T, Amos S, Agmon O, et al. Is it me or my hormones? Neuroendocrine activation profiles to visual food stimuli across the menstrual cycle. J Clin Endocrinol Metab. 2017;102(9):3406-14. https://doi.org/10.1210/jc.20163921.

36. Scheele D, Plota J, Stoffel-Wagner B, Maier W, Hurlemann R. Hormonal contraceptives suppress oxytocin-induced brain reward responses to the partner's face. Soc Cogn Affect Neurosci. 2016;11(5):767-74. https://doi.org/10.1093/scan/nsv157.

37. Jakob K, Ehrentreich H, Holtfrerich SKC, Reimers L, Diekhof EK. DAT1-Genotype and menstrual cycle, but not hormonal contraception, modulate reinforcement learning: preliminary evidence. Front Endocrinol (Lausanne). 2018;9:60. https://doi.org/10.3389/fendo. 2018.00060. The authors show a possible underlying neurobiological mechanism of how estradiol may impact reward processing in women using a longitudinal study design. Although the influence of $\mathrm{HC}$-induced changes in endogenous and exogenous estradiol levels on dopamine neurotransmission remains to be investigated, this study provides first hypotheses.
38. Merz CJ. Contribution of stress and sex hormones to memory encoding. Psychoneuroendocrinology. 2017;82:51-8. https://doi. org/10.1016/j.psyneuen.2017.05.002.

39. Barel E, Abu-Shkara R, Colodner R, Masalha R, Mahagna L, Zemel OC, et al. Gonadal hormones modulate the HPA-axis and the SNS in response to psychosocial stress. J Neurosci Res. 2018;96(8):1388-97. https://doi.org/10.1002/jnr.24259.

40. Nielsen SE, Ahmed I, Cahill L. Postlearning stress differentially affects memory for emotional gist and detail in naturally cycling women and women on hormonal contraceptives. Behav Neurosci. 2014;128(4):482-93. https://doi.org/10.1037/a0036687.

41. Mordecai KL, Rubin LH, Eatough E, Sundermann E, Drogos L, Savarese A, et al. Cortisol reactivity and emotional memory after psychosocial stress in oral contraceptive users. J Neurosci Res. 2017;95(1-2):126-35. https://doi.org/10.1002/jnr.23904.

42.• Hertel J, Konig J, Homuth G, Van der Auwera S, Wittfeld K, Pietzner M, et al. Evidence for stress-like alterations in the hpaaxis in women taking oral contraceptives. Sci Rep. 2017;7(1): 14111. https://doi.org/10.1038/s41598-017-13927-7. The authors investigated different types of physiological as well as structural MRI data in a large cohort $(N=233)$. Thereby, they provide integrative results on $\mathrm{OC}$-effects on stress response of the HPAaxis linked with morphological alterations in hippocampal gray matter.

43. Harmer CJ. Emotional processing and antidepressant action. Curr Top Behav Neurosci. 2013;14:209-22. https://doi.org/10.1007/ 7854_2012_210.

44. Warren MB, Pringle A, Harmer CJ. A neurocognitive model for understanding treatment action in depression. Philos Trans R Soc Lond Ser B Biol Sci. 2015;370(1677):20140213. https://doi.org/10. 1098/rstb.2014.0213.

45. Godlewska BR, Browning M, Norbury R, Cowen PJ, Harmer CJ. Early changes in emotional processing as a marker of clinical response to SSRI treatment in depression. Transl Psychiatry. 2016;6(11):e957. https://doi.org/10.1038/tp.2016.130.

46. Adolphs R. The neurobiology of social cognition. Curr Opin Neurobiol. 2001;11(2):231-9.

47. Joiner TE, Timmons KA. Depression in its interpersonal context. In: Gotlib IH, Hammen CL, editors. Hanbook of depression. New York: Guilford Press; 2009. p. 322-39.

48. Fieker M, Moritz S, Kother U, Jelinek L. Emotion recognition in depression: an investigation of performance and response confidence in adult female patients with depression. Psychiatry Res. 2016;242:226-32. https://doi.org/10.1016/j.psychres.2016.05.037.

49. Davidson RJ. Affective style and affective disorders: perspectives from affective neuroscience. Cognition Emotion. 1998;12(3):30730. https://doi.org/10.1080/026999398379628.

50. Sontag LM, Graber JA. Coping with perceived peer stress: genderspecific and common pathways to symptoms of psychopathology. Dev Psychol. 2010;46(6):1605-20. https://doi.org/10.1037/ a0020617.

51. Shapero BG, Abramson LY, Alloy LB. Emotional reactivity and internalizing symptoms: moderating role of emotion regulation. Cognit Ther Res. 2016;40(30):328-40. https://doi.org/10.1007/ s10608-015-9722-4.

52. McLaughlin KA, Kubzansky LD, Dunn EC, Waldinger R, Vaillant G, Koenen KC. Childhood social environment, emotional reactivity to stress, and mood and anxiety disorders across the life course. Depress Anxiety. 2010;27(12):1087-94. https://doi.org/10.1002/ da.20762.

53. Montague PR, Dolan RJ, Friston KJ, Dayan P. Computational psychiatry. Trends Cogn Sci. 2012;16(1):72-80. https://doi.org/10. 1016/j.tics.2011.11.018.

54. Huys QJ, Daw ND, Dayan P. Depression: a decision-theoretic analysis. Annu Rev Neurosci. 2015;38:1-23. https://doi.org/10.1146/ annurev-neuro-071714-033928. 
55. Eldar E, Rutledge RB, Dolan RJ, Niv Y. Mood as representation of momentum. Trends Cogn Sci. 2016;20(1):15-24. https://doi.org/ 10.1016/j.tics.2015.07.010.

56. Diekhof EK, Ratnayake M. Menstrual cycle phase modulates reward sensitivity and performance monitoring in young women: preliminary fMRI evidence. Neuropsychologia. 2016;84:70-80. https://doi.org/10.1016/j.neuropsychologia.2015.10.016.

57. Reimers L, Buchel C, Diekhof EK. How to be patient. The ability to wait for a reward depends on menstrual cycle phase and feedbackrelated activity. Front Neurosci. 2014;8:401. doi:https://doi.org/10. 3389/fnins.2014.00401.

58. Dreher JC, Schmidt PJ, Kohn P, Furman D, Rubinow D, Berman KF. Menstrual cycle phase modulates reward-related neural function in women. Proc Natl Acad Sci U S A. 2007;104(7):2465-70. https://doi.org/10.1073/pnas.0605569104.

59. Ossewaarde L, van Wingen GA, Kooijman SC, Backstrom T, Fernandez G, Hermans EJ. Changes in functioning of mesolimbic incentive processing circuits during the premenstrual phase. Soc Cogn Affect Neurosci. 2011;6(5):612-20. https://doi.org/10.1093/ scan/nsq071.

60. O'Doherty JP. Reward representations and reward-related learning in the human brain: insights from neuroimaging. Curr Opin Neurobiol. 2004;14(6):769-76. https://doi.org/10.1016/j.conb. 2004.10.016.

61. Elliott R, Dolan RJ, Frith CD. Dissociable functions in the medial and lateral orbitofrontal cortex: evidence from human neuroimaging studies. Cereb Cortex. 2000;10(3):308-17.

62. Shams WM, Cossette MP, Shizgal P, Brake WG. 17beta-estradiol locally increases phasic dopamine release in the dorsal striatum. Neurosci Lett. 2018;665:29-32. https://doi.org/10.1016/j.neulet. 2017.11.039.

63. Yoest KE, Cummings JA, Becker JB. Estradiol, dopamine and motivation. Cent Nerv Syst Agents Med Chem. 2014;14(2):83-9.

64. Diekhof EK. Be quick about it. Endogenous estradiol level, menstrual cycle phase and trait impulsiveness predict impulsive choice in the context of reward acquisition. Horm Behav. 2015;74:186-93. https://doi.org/10.1016/j.yhbeh.2015.06.001.

65. Smith CT, Sierra Y, Oppler SH, Boettiger CA. Ovarian cycle effects on immediate reward selection bias in humans: a role for estradiol. J Neurosci. 2014;34(16):5468-76. https://doi.org/10.1523/ JNEUROSCI.0014-14.2014.

66. Diekhof EK. Estradiol and the reward system in humans. Curr Opin Behav Sci. 2018;23:58-64. https://doi.org/10.1016/j.cobeha.2018. 03.010.

67. Albert KM, Newhouse PA. Estrogen, stress, and depression: cognitive and biological interactions. Annu Rev Clin Psychol. 2019;15: 399-423. https://doi.org/10.1146/annurev-clinpsy-050718095557. Extensive literature review on interactions of endogenous estrogens, stress, and depression. The authors comprehensively explain biological and cognitive mechanisms underlying stress response and provide a model of estradiol's influence on these mechanisms across the menstrual cycle and during menopause.

68. Jacobs EG, Holsen LM, Lancaster K, Makris N, Whitfield-Gabrieli $\mathrm{S}$, Remington A, et al. 17beta-estradiol differentially regulates stress circuitry activity in healthy and depressed women. Neuropsychopharmacology. 2015;40(3):566-76. https://doi.org/ 10.1038/npp.2014.203.

69. Hodes GE, Epperson CN. Sex differences in vulnerability and resilience to stress across the life span. Biol Psychiatry. 2019;86:42132. https://doi.org/10.1016/j.biopsych.2019.04.028.

70. Snyder JS, Soumier A, Brewer M, Pickel J, Cameron HA. Adult hippocampal neurogenesis buffers stress responses and depressive behaviour. Nature. 2011;476(7361):458-61. https://doi.org/10. 1038/nature10287.
71. Holsboer F. The corticosteroid receptor hypothesis of depression. Neuropsychopharmacology. 2000;23(5):477-501. https://doi.org/ 10.1016/S0893-133X(00)00159-7.

72. Aleknaviciute J, Tulen JHM, De Rijke YB, Bouwkamp CG, van der Kroeg M, Timmermans M, et al. The levonorgestrel-releasing intrauterine device potentiates stress reactivity. Psychoneuroendocrinology. 2017;80:39-45. https://doi.org/10. 1016/j.psyneuen.2017.02.025. Cross-sectional study that provides insight into how different $\mathrm{HC}$ methods, in this case LNG-IUD vs. OC, may have a different impact on endocrine stress responsivity. The authors found that IUDs alter systemic physiological responses by leaking a considerable amount of progestin into the systemic circulation, which might influence mood.

73. Pekin AT, Kerimoglu OS, Kebapcilar AG, Yilmaz SA, Benzer N, Celik C. Depressive symptomatology and quality of life assessment among women using the levonorgestrel-releasing intrauterine system: an observational study. Arch Gynecol Obstet. 2014;290(3): 507-11. https://doi.org/10.1007/s00404-014-3237-1.

74. Morotti E, Casadio P, Guasina F, Battaglia B, Mattioli M, Battaglia C. Weight gain, body image and sexual function in young patients treated with contraceptive vaginal ring. A prospective pilot study. Gynecol Endocrinol. 2017;33(8):660-4. https://doi.org/10.1080/ 09513590.2017 .1306850

75. Jain S, Vaid NB, Narang Y, Suneja A, Guleria K. A randomised controlled trial comparing the efficacy and side-effects of intravaginal ring (Nuvaring $((\mathrm{R}))$ ) with combined oral hormonal preparation in dysfunctional uterine bleeding. J Clin Diagn Res. 2016;10(3):QC21-4. https://doi.org/10.7860/JCDR/2016/16545. 7516.

76. Lopez LM, Grimes DA, Gallo MF, Stockton LL, Schulz KF. Skin patch and vaginal ring versus combined oral contraceptives for contraception. Cochrane Database Syst Rev. 2013;4:CD003552. https://doi.org/10.1002/14651858.CD003552.pub4.

77. Worly BL, Gur TL, Schaffir J. The relationship between progestin hormonal contraception and depression: a systematic review. Contraception. 2018;97(6):478-89. https://doi.org/10.1016/j. contraception.2018.01.010. Systematic review on the association between progestin-only HCs and depression, paying particular attention to the different modes of $\mathrm{HC}$ administration used in each study. The authors conclude that there is only minimal association between progestin-only HCs and depression. The authors discuss the common pitfalls and different risks of bias observed across the reviewed studies.

78. Pardridge WM, Mietus LJ. Transport of steroid hormones through the rat blood-brain barrier. Primary role of albumin-bound hormone. J Clin Invest. 1979;64(1):145-54. https://doi.org/10.1172/ JCI109433.

79. Kim H, Yu T, Cam-Etoz B, van Groen T, Hubbard WJ, Chaudry IH. Treatment of traumatic brain injury with 17alpha-ethinylestradiol3-sulfate in a rat model. J Neurosurg. 2017;127(1):23-31. https:// doi.org/10.3171/2016.7.JNS161263.

80. Pike VW. PET radiotracers: crossing the blood-brain barrier and surviving metabolism. Trends Pharmacol Sci. 2009;30(8):431-40. https://doi.org/10.1016/j.tips.2009.05.005.

81. Barkhem T, Carlsson B, Nilsson Y, Enmark E, Gustafsson J-Å, Nilsson SJMp. Differential response of estrogen receptor $\alpha$ and estrogen receptor $\beta$ to partial estrogen agonists/antagonists 1998;54(1):105-112.

82. Jones EF, Ray KM, Li W, Chien AJ, Mukhtar RA, Esserman LJ et al. Initial experience of dedicated breast PET imaging of ER+ breast cancers using [F-18] fluoroestradiol. 2019;5(1):12.

83. van Kruchten M, de Vries EG, Brown M, de Vries EF, Glaudemans AW, Dierckx RA et al. PET imaging of oestrogen receptors in patients with breast cancer. 2013;14(11):e465-ee75. 
84. Moresco R, Casati R, Lucignani G, Carpinelli A, Schmidt K, Todde S, et al. Systemic and cerebral kinetics of $16 \alpha[18 \mathrm{~F}]$ Fluoro-17 $\beta$ estradiol: a ligand for the in vivo assessment of estrogen receptor binding. Parameters. 1995;15(2):301-11.

85. Khayum MA, de Vries EF, Glaudemans AW, Dierckx RA, Doorduin JJJoNN. In vivo imaging of brain estrogen receptors in rats: a $16 \alpha-18$ F-fluoro-17 $\beta$-estradiol PET study. 2014;55(3):481487.

86. Hattersley G, David F, Harris A, Clarkin M, Banks K, Glaudemans AWJM, et al. A phase 1 dose escalation study of RAD1901, an oral selective estrogen receptor degrader, in healthy postmenopausal women. Cancer Res. 2016;76. https://doi.org/10.1158/1538-7445. Sabcs15-P6-13-02.

87. Moraga-Amaro R, Van Waarde A, Doorduin J, De Vries EJJon. Sex steroid hormones and brain function: PET imaging as a tool for research 2018;30(2):e12565.
88. Lee JH, Zhou H-b, Dence CS, Carlson KE, Welch MJ, Katzenellenbogen JAJBc. Development of [F-18] fluorinesubstituted tanaproget as a progesterone receptor imaging agent for positron emission tomography. 2010;21(6):1096-104.

89. Sanders SA, Graham CA, Bass JL, Bancroft J. A prospective study of the effects of oral contraceptives on sexuality and well-being and their relationship to discontinuation. Contraception. 2001;64(1): $51-8$.

90. Lindh I, Blohm F, Andersson-Ellstrom A, Milsom I. Contraceptive use and pregnancy outcome in three generations of Swedish female teenagers from the same urban population. Contraception. 2009;80(2):163-9. https://doi.org/10.1016/j.contraception.2009. 01.019 .

Publisher's Note Springer Nature remains neutral with regard to jurisdictional claims in published maps and institutional affiliations. 